Proceedings of the 1996 IEEE

International Conference on Robotics and Automation

Minneapolis, Minnesota - April 1996

\title{
Creep Dynamics of Nonholonomic Systems
}

\author{
Jiunn-Cherng Wang, Han-Pang Huang \\ Department of Mechanical Engineering, National Taiwan University \\ Taipei, 10674 TAIWAN, R.O.C.
}

\begin{abstract}
Basic concepts about the creep behavior of nonholonomic constrained (NC) systems were discussed in [5]. Two fundamental types of creep kinematics were proposed and the hybrid ones could be made. In this work, we extend to the creep dynamics of typical NC systems such as the disk, sleigh and wheel. First, certain reductive models of constrained motion such as ideal, relative, pair, and general-pair models are explored and related. Secondly, by the invariant manifold method of singular perturbation, fundamental rotational and traverse creep dynamics and a hybrid one are calculated in an approximate way. They can be close to the complete system as possible. Therefore, the bridge between reductive and invariant analyses can be made by the model of general-pair creep and can help us understand physical implications behind the approximate solution$s$. It is proven that advanced vehicle techniques, such as the anti-lock braking system and a special tracking control system, can be realized by the proposed quasi constrained creeps.
\end{abstract}

\section{Introduction}

The dynamical formulation of quasi constrained motion [6] for typical NC systems is represented here by two fundamental creep models been described in [5]. It is understood that creeps arise from the natural sources of viscous frictions [2]. Relaxations are allowed against the constraints to certain extent. Namely, the infinitesimal movements are pseudoly rather than absolutely restricted. Even that, the governed equations of motion can be still reduced. Namely, original constraint manifold can be converted into a quasi one. In spite it is not easy to obtain directly, we could find an approximate one.

For a constrained dynamical description, there are usually two ways to formulate it. One is the $L a$ grange multiplier method, the other is the elimination of dependent variables. The former can be handled by various algebraic techniques [1] and can get the constrained force directly. The latter is a reductive scheme and has more analytical implications [3]. Our quasi analysis belongs to the latter. We first explore certain specific kinematical motions such as the ideal, relative, pair and general-pair models. They will be related to the subsequent construction of an approximated quasi manifold.

By the invariant manifold method of singular perturbation [4], the creep dynamics of various order approximation can be obtained as long as the viscosity parameter of friction is sufficiently large. Therefore, quasi wheels can be governed by a model of creep of general-pair, which bridges the specifically reduced and the singularly perturbed schemes.

In spite many studies [3] focused on the nonideally constrained problems, this is the first time, to our best knowledge, to make use of creep models in the dynamical formulation of NC systems. From the analytical derivation, the creep model can be recognized as a modification of those ideal results. Fig. 1 shows a half-saturation friction model and a creep model. Note that only the small linear regions are considered. Friction force $f$ is proportional to the normal speed $v_{N}$ with slope $b . v_{N_{0}}$ and $e_{0}$ are respectively an offset value and a constant slope to tangential speed $v_{T}$.

Creep has much practical sense. Advanced vehicle techniques, such as the anti-lock braking system (AB$\mathrm{S})$ and a special tracking control system (TCS), can be proven feasible.

\section{Fact (Common NC Motions)}

Most NC systems have the common motions in Fig. 2 with rolling ( $\mathrm{r}$ ), longitudinal translating (l) and $z$ axis orienting $(\mathrm{z})$. The traverse motion $(\mathrm{t})$ is often limited. In fact, case (a), (b) and (c) are motions of 
disk, sleigh and wheel respectively.
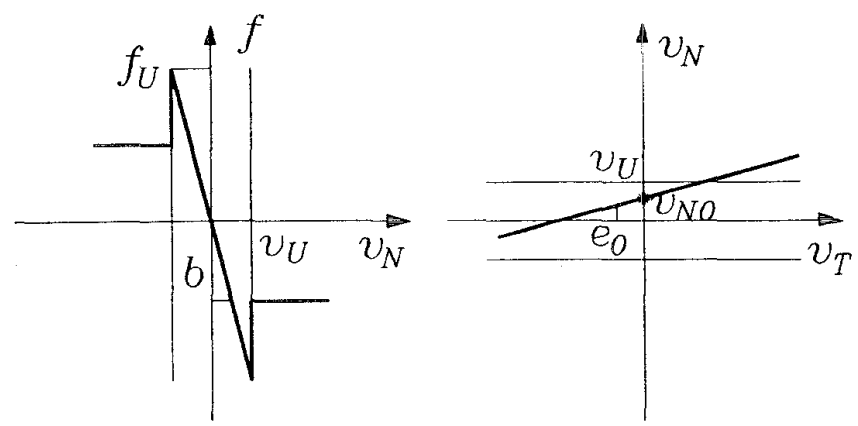

Fig. 1 Friction and creep models of quasi NC systems.

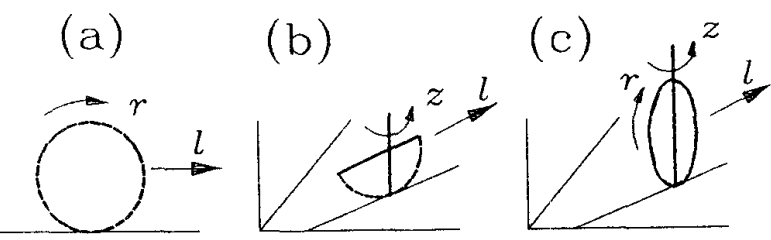

Fig. 2 Common nonholonomic motions.

\section{Rotational Creep Dynamics}

Consider the 1D rolling disk shown in Fig. 3. It has mass $m$, polar inertia $J$, radius $r$ and subjects to external forces $E_{d}=\left[E_{l}, E_{r}\right]^{t}$ and control forces $u_{d}=\left[u_{l}, u_{r}\right]^{t}$. The disk motion can be expressed by the derivative of longitudinal speed $v_{l}$ and rotational angular speed $\omega_{r}, v_{d}=\left[v_{l}, \omega_{r}\right]^{t}$,

$$
D_{d} \dot{v}_{d}=E_{d}+f_{d}+u_{d}
$$

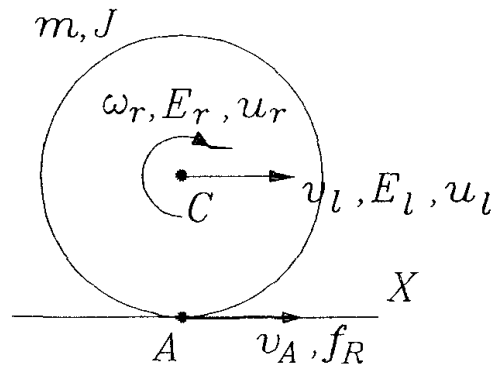

Fig. 3 Dynamic model of disk.

where $D_{d}=\operatorname{diag}(m, J) ; f_{d}=\left[f_{R},-r f_{R}\right]^{t} ; f_{R}$ denotes the constraint force acted at contact point $A$. External forces, such as air and bearing resistance, are dissipative $E_{l}, E_{r}$ with parameters $h_{l}, h_{r}$,

$$
E_{l}=-h_{l} v_{l}, \quad E_{r}=-h_{r} \omega_{r}
$$

Control forces $u_{l}$ and $u_{r}$ are considered constant here for the dynamical discussion.

The constrained reaction belongs to tractive force occurred from the horizontal line. The pseudoviolated behavior appears whenever $f_{R}$ arises from the viscous friction,

$$
f_{R}=-b_{R} v_{A}, \text { for }\left|v_{A}\right|<v_{A U},\left|f_{R}\right|<f_{R U} .
$$

Note that $b_{R}$ is a sufficiently large parameter. The slipping and skidding are not taken into account here.

Let us introduce the dimensionless coordinates, originated from [3] and extended here, with a subindex ' 0 ',

$$
\begin{gathered}
x_{0}=\frac{x}{r}, \quad t_{0}=\frac{h_{l} t}{m}, \quad f_{R 0}=\frac{m f_{R}}{r h_{l}^{2}}, \quad u_{l 0}=\frac{m u_{l}}{r h_{l}^{2}}, \\
u_{r 0}=\frac{m u_{r}}{r^{2} h_{l}^{2}}, \quad \alpha_{J}=\frac{J}{m r^{2}}, \quad \beta_{r}=\frac{h_{r}}{r^{2} h_{l}}, \quad \gamma_{R}=\frac{b_{R}}{h_{l}}
\end{gathered}
$$

and write $v_{l 0}=x_{0}^{\prime}=d x_{0} / d t_{0}, \omega_{r 0}=\theta^{\prime}=d \theta / d t_{0}$. The relations of velocity, acceleration and Lagrangian are respectively $v_{l 0}=m v_{l} /\left(r h_{l}\right), v_{l 0}^{\prime}=m^{2} \dot{v}_{l} /\left(r h_{l}^{2}\right), L_{0}=$ $m L /\left(r^{2} h_{l}^{2}\right)$, etc... Note that the creep coefficients are dimensionless themselves. Hence, the constraint becomes $v_{10}-\omega_{r 0}-v_{A 0}=0$ and the Lagrangian of the disk equals to $L_{0}=\left(v_{l 0}^{2}+\alpha_{J} \omega_{r 0}^{2}\right) / 2$. Complete equations can be re-written as

$$
\begin{gathered}
v_{l 0}^{\prime}=-\left(1+\gamma_{R}\right) v_{l 0}+\gamma_{R} \omega_{r 0}+u_{l 0} \\
\left.\alpha_{J} \omega_{r 0}^{\prime}=\gamma_{R} v_{l 0}-\left(\beta_{r}+\gamma_{R}\right) \omega_{r 0}+u_{r 0} \text { (complete) }\right)_{0}
\end{gathered}
$$

with friction $f_{R 0}=-\gamma_{R} v_{A 0}$.

\section{Theorem (Specifically Kinematical Motions)}

When the instantaneous speed $v_{A 0}$ at the contact point of the disk is specified as (i) zero (ideal), or (ii) a constant value $v_{A 1}$ (relative), or (iii) proportional to the rotational angular speed $\omega_{r 0}$ (pair), or (iv) a constant $v_{A 1}$ plus a linear $\omega_{r 0}$ term (general-pair), the disk motion can be expressed by using only one differential equation together with this kinematic relation. [1]

The governed equations of ideal (i) and general-pair (iv) cases are shown below

$$
\begin{aligned}
& \left(1+\alpha_{J}\right) \omega_{r 0}^{\prime}=-\left(1+\beta_{r}\right) \omega_{r 0}+u_{10} \quad \text { (ideal) (6) } \\
& \left(1+\alpha_{J e}\right) \omega_{r 0}^{\prime}=-v_{A 1}-\left(1+\beta_{r e}\right) \omega_{r 0}+u_{10} .
\end{aligned}
$$


Cases (ii)-(iv) explicitly depict a specific constraint manifold about the pseudo violated disk, which characterizes a slight modification of the ideal manifold $M_{d}$ in case (i). The ideal case can be proven valid [2] for a free disk rolling as the parameter $\gamma_{R}$ approaches infinity since $v_{\mathcal{A} 1}$ and $e_{r 0}$ approach zeros.

\section{Corollary (Quasi Constraint Manifold)}

If there exists a generally kinematical relation between the instantaneous speed $v_{A 0}$ and angular speed $\omega_{r 0}$,

$$
v_{A 0}=g_{d}\left(\omega_{r 0}\right), \quad g_{d} \in \mathcal{C}^{1} \text { (continuous) }
$$

only one differential equation is required.

(2) can be recognized as a real quasi constraint manifold $\mathcal{M}_{d}$ of disk, which is difficult to accurately obtain. However, a simplified manifold $\tilde{\mathcal{M}}_{d}$ can be approximated by the specific motion of (7) with suitable parameters $\tilde{v}_{A 1}, \tilde{e}_{r 0}$. This will be explained later.

\section{Theorem (Rotational Creep Dynamics)}

The quasi constrained disk can be approximated by the rotational creep dynamics described by only one differential equation as long as the viscous coefficient $\gamma_{\boldsymbol{R}}$ is sufficiently large. Such approximation can be as close as possible whenever it is needed.

(proof): Firstly, take a small parameter $\epsilon=1 / \gamma_{R}$ and transfer (5) into a singularly perturbed form

$$
\begin{gathered}
\epsilon v_{A 0}^{\prime}=-\left(\epsilon+1+\frac{1}{\alpha_{J}}\right) v_{A 0}+\left(\frac{\beta_{\tau}}{\alpha_{J}}-1\right) \epsilon \omega_{r 0}+\frac{\epsilon u_{20}}{\alpha_{J}} \\
\omega_{r 0}^{\prime}=\frac{v_{A 0}}{\epsilon \alpha_{J}}-\frac{\beta_{r} \omega_{r 0}}{\alpha_{J}}+\frac{u_{r 0}}{\alpha_{J}} . \quad \text { (singular) }
\end{gathered}
$$

When $\epsilon=0\left(\gamma_{R}=\infty\right),(9 \mathrm{a})$ gives $v_{A 0}=0$ and $(9 \mathrm{~b})$ becomes identical to the ideal (6). When $\gamma_{R}$ is finite and sufficiently large, assume that the quasi constraint manifold $\mathcal{M}_{d}$ in (8) can be expressed as a power series form,

$$
v_{A 0}=g_{R}\left(\omega_{r 0}, \epsilon\right)=g_{R 0}+\epsilon g_{R 1}+\epsilon^{2} g_{R 2}+\cdots .
$$

By the invariant manifold method [4], (10) is plugged into (9) and obtains the PDE, condition,

$$
\begin{gathered}
\epsilon\left(\frac{\partial g_{R 0}}{\partial \omega_{r 0}}+\epsilon \frac{\partial g_{R 1}}{\partial \omega_{r 0}}+\epsilon^{2} \frac{\partial g_{R 2}}{\partial \omega_{r 0}}+\cdots\right) \\
\left(\frac{g_{R 0}+\epsilon g_{R 1}+\epsilon^{2} g_{R 2}+\cdots}{\epsilon \alpha_{J}}-\frac{\beta_{r} \omega_{r 0}}{\alpha_{J}}+\frac{u_{r 0}}{\alpha_{J}}\right)=-\left(\epsilon+1+\frac{1}{\alpha_{J}}\right) \\
\left(g_{R 0}+\epsilon g_{R 1}+\epsilon^{2} g_{R 2}+\cdots\right)+\left(\frac{\beta_{r}}{\alpha_{J}}-1\right) \epsilon \omega_{r 0}+\frac{\epsilon u_{20}}{\alpha_{J}} .
\end{gathered}
$$

Equating the expanded $\epsilon$ coefficients, it is found

$$
\begin{array}{r}
g_{R 0}=0, \quad g_{R 1}=\frac{-\beta_{1} \omega_{r 0}+u_{20}}{1+\alpha_{J}}, \\
g_{R 2}=\frac{\alpha_{J}\left(\beta_{1}^{2} \omega_{r 0}-\beta_{r} u_{10}-u_{30}\right)}{\left(1+\alpha_{J}\right)^{3}}
\end{array}
$$

where $\beta_{1}=\alpha_{J}-\beta_{r}$ and $u_{30}=\alpha_{J}^{2} u_{l 0}-2 \alpha_{J} u_{r 0}-u_{r 0}$. $g_{R 0}=0$, is consistent with the ideal disk. We also notice that the linear $\omega_{r}$ terms occur in $g_{R 1}$ and $g_{R 2}$.

By ignoring high order $(>2)$ terms, the 1st and 2nd order approximated speeds equal to $\tilde{v}_{A 0}^{1}=\epsilon g_{R 1}$, $\tilde{v}_{A 0}^{2}=\epsilon g_{R 1}+\epsilon^{2} g_{R 2}$. Substituting $\tilde{v}_{A 0}^{1}$ into $(9 \mathrm{~b})$, the resultant 1 st order creep equation (denoted by creep1) happens identical to the ideal (6). But, the speed $\tilde{v}_{A 0}^{1}$ does not vanish any more. If $\tilde{v}_{A 0}^{2}$ is taken into account, the 2nd order creep dynamics can be obtained as

$\omega_{r 0}^{\prime}=\frac{-\left(1+\beta_{r}\right) \omega_{r 0}+u_{10}}{1+\alpha_{J}}+\frac{\epsilon\left(\beta_{1}^{2} \omega_{r 0}-\beta_{r} u_{10}-u_{30}\right)}{\left(1+\alpha_{J}\right)^{3}}$.

(creep2) (13)

\section{Corollary (Creep Dynamics of General-Pair)}

Quasi constrained disk can be approximated by the creep of general-pair motion like (7) with suitable 1st order and 2 nd order replaced parameters, denoted by cgp1 and cgp2 respectively,

$$
\begin{gathered}
\tilde{v}_{A 1}^{1}=\frac{\epsilon u_{20}}{1+\alpha_{J}}, \quad \tilde{e}_{r 0}^{1}=\frac{\epsilon\left(\alpha_{J}-\beta_{r}\right)}{1+\alpha_{J}} \quad(\operatorname{cgp} 1) \\
\tilde{v}_{A 1}^{2}=\frac{\epsilon u_{20}}{1+\alpha_{J}}-\frac{\epsilon^{2} \alpha_{J}\left(\beta_{r} u_{10}+u_{30}\right)}{\left(1+\alpha_{J}\right)^{3}}, \\
\tilde{e}_{r 0}^{2}=\frac{\epsilon\left(\alpha_{J}-\beta_{r}\right)}{1+\alpha_{J}}-\frac{\epsilon^{2} \alpha_{J}\left(\alpha_{J}-\beta_{r}\right)^{2}}{\left(1+\alpha_{J}\right)^{3}} . \quad(\operatorname{cgp} 2)
\end{gathered}
$$

The above corollary gives a bridge between reductive specific and invariant analyses. Eq. (14) can be obtained directly by equating $\tilde{v}_{A 0}^{i}=\tilde{v}_{A 1}^{i}-\tilde{e}_{r 0}^{i} \omega_{r 0}, i=$ 1,2 . Note that the creep dynamics is a form of singular perturbation and the 'cgp' ones are forms of specifically kinematical motions. We also notice from (14) that the constant inputs directly affect the $\tilde{v}_{A 1}^{i}$ and only viscous parameters occur in the $\tilde{e}_{r 0}^{i}$.

\section{Traverse Creep Dynamics}

Consider the sleigh shown in Fig. 4 with speeds $\left[v_{l}, v_{t}, \omega_{z}\right]^{t}$ of contact point $A$ along the moving frame 
$\mathcal{F}(A x y z)$

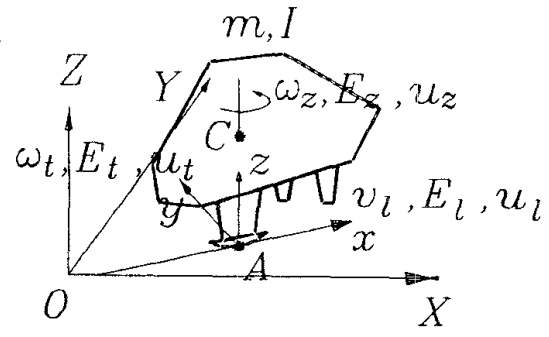

Fig. 4 Dynamic model of sleigh.

The mass and inertia of sleigh are $m$ and $I$ with a reference length $r$. Under the external force $\left[E_{l}, E_{t}, E_{z}\right]^{t}$ and constant control $\left[u_{l}, u_{t}, u_{z}\right]^{t}$, the sleigh evolves as

$$
\begin{gathered}
v_{l 0}^{\prime}=v_{t 0} \omega_{z 0}-v_{l 0}+u_{l 0} \\
v_{t 0}^{\prime}=-v_{10} \omega_{z 0}-\left(\beta_{t}+\gamma_{T}\right) v_{t 0}+u_{t 0} \\
\left.\alpha_{I} \omega_{z 0}^{\prime}=-\beta_{z} \omega_{z 0}+u_{z 0} \quad \text { (complete) }\right)_{0}
\end{gathered}
$$

with friction $f_{T 0}=-\gamma_{T} v_{t 0}$. Notice that the orienting behavior is uncoupled. Additional dimensionless relations are used in (15),

$$
\alpha_{I}=\frac{I}{m r^{2}}, \quad \beta_{t}=\frac{h_{t}}{h_{l}}, \quad \beta_{z}=\frac{h_{z}}{r^{2} h_{l}}, \quad \gamma_{T}=\frac{b_{T}}{h_{l}} .
$$

Specifically kinematical motions of sleigh can be derived. For a general-pair motion $\left(v_{t 0}=v_{t 1}+e_{t 0} v_{70}\right)$, only two differential equations are needed,

$$
v_{l 0}^{\prime}=\left(v_{t 1}+e_{t 0} v_{l 0}\right) \omega_{z 0}-v_{l 0}+u_{l 0} \text { (general-pair) }
$$

where the orienting component remains the same as preceding. At this time, true creep coefficient is equal to $e_{t}=e_{t 0}+v_{t 1} / v_{l 0}$.

The 1st and 2nd order traverse creep dynamics of sleigh can be obtained as

$$
\begin{aligned}
& \left.v_{l 0}^{\prime}=\epsilon \omega_{z 0}\left(u_{t 0}-v_{l 0} \omega_{z 0}\right)-v_{l 0}+u_{l 0} \quad \text { (creep } 1\right)(18) \\
& v_{l 0}^{\prime}=\epsilon \omega_{z 0}\left\{u_{t 0}-v_{l 0} \omega_{z 0}+\epsilon\left[\left(\beta_{t}-1-\frac{\beta_{z}}{\alpha_{I}}\right) v_{l 0} \omega_{z 0}+\right.\right. \\
& \left.\left.u_{l 0} \omega_{z 0}-\beta_{t} u_{t 0}+\frac{u_{z 0} v_{l 0}}{\alpha_{I}}\right]\right\}-v_{l 0}+u_{l 0} . \quad \text { (creep2) }
\end{aligned}
$$

Since the orientation is uncoupled, a desired value, $\omega_{z 0}^{d}$, can be easily assumed. Therefore, the generalpair creep motion of sleigh can be derived as (17) with the following parameters,

$$
\begin{gathered}
\tilde{v}_{t 1}^{1}=\epsilon u_{t 0}, \quad \tilde{e}_{t 0}^{1}=-\epsilon \omega_{z 0}^{d} \quad(\operatorname{cgp} 1)(20 \mathrm{a}) \\
\tilde{v}_{t 1}^{2}=\epsilon u_{t 0}+\epsilon^{2}\left(u_{i 0} \omega_{z 0}^{d}-\beta_{t} u_{t 0}\right), \\
\tilde{e}_{t 0}^{2}=-\epsilon \omega_{z 0}^{d}+\epsilon^{2}\left[\left(\beta_{t}-1-\frac{\beta_{z}}{\alpha_{I}}\right) \omega_{z 0}^{d}+\frac{u_{z 0}}{\alpha_{I}}\right] . \quad(\operatorname{cgp} 2)(20 \mathrm{~b})
\end{gathered}
$$

\section{Hybrid Creep Dynamics}

A single wheeled vehicle with mass $m$, equatorial inertia $I$ and polar inertia $J$ on a plane is depicted in Fig. 5. When external force $\left[E_{l}, E_{t}, E_{z}, E_{\mathrm{r}}\right]^{T}$ and control $\left[u_{l}, u_{t}, u_{t}, u_{r}\right]^{t}$ are applied, the complete governed equations are

$$
\begin{gathered}
v_{l 0}^{\prime}=v_{t 0} \omega_{z 0}-\left(1+\gamma_{R}\right) v_{l 0}+\gamma_{R} \omega_{r 0}+u_{l 0} \\
v_{t 0}^{\prime}=-v_{l 0} \omega_{z 0}-\left(\beta_{t}+\gamma_{T}\right) v_{t 0}+u_{t 0} \\
\alpha_{I} \omega_{z 0}^{\prime}=-\beta_{z} \omega_{z 0}+u_{z 0} \\
\alpha_{J} \omega_{r 0}^{\prime}=\gamma_{R} v_{l 0}-\left(\beta_{r}+\gamma_{R}\right) \omega_{r 0}+u_{r 0} .(\text { complete })_{0}(21) \\
\text { with frictions } f_{R 0}=-\gamma_{R} v_{A 0}=-\gamma_{R}\left(v_{l 0}-\omega_{r 0}\right), f_{T 0}= \\
-\gamma_{T} v_{t 0} .
\end{gathered}
$$

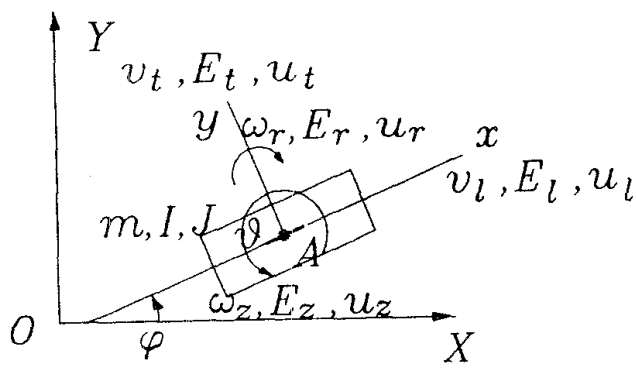

Fig. 5 Dynamic model of wheel.

By the similar procedure, derivation results are depicted below. First, the general-pair motion model of wheel is derived as $\left(v_{A 0}=v_{A 1}-e_{r 0} \omega_{r 0}, v_{t 0}=\right.$ $\left.v_{t 1}+e_{t 0}\left[v_{A 1}+\left(1-e_{r 0}\right) \omega_{r 0}\right]\right)$,

$$
\begin{array}{r}
\left(1+\alpha_{J e}\right) \omega_{r 0}^{\prime}=\left\{v_{t 1}+e_{t 0}\left[v_{A 1}+\left(1-e_{r 0}\right) \omega_{r 0}\right]\right\} \omega_{z 0}-v_{A 1} \\
-\left(1+\beta_{r e}\right) \omega_{r 0}+u_{10} . \quad \text { (general-pair) (22) }
\end{array}
$$

The second one is the hybrid creep dynamics of second order,

$$
\begin{gathered}
\omega_{r 0}^{\prime}=\frac{-\left(1+\beta_{r}\right) \omega_{r 0}+u_{10}+\epsilon \omega_{z 0}\left(u_{t 0}-\omega_{z 0} \omega_{r 0}\right) / b}{1+\alpha_{J}} \\
+\frac{\epsilon\left[\left(\alpha_{J}-\beta_{r}\right)^{2} \omega_{r 0}-\beta_{r} u_{10}-u_{30}\right]}{\left(1+\alpha_{J}\right)^{3}} . \quad(\text { creep } 2)(23)
\end{gathered}
$$

The third is the general-pair creep model of wheel with parameters :

$$
\begin{aligned}
& \tilde{v}_{A 1}^{1}=\frac{\epsilon u_{20}}{1+\alpha_{J}}, \quad \tilde{e}_{r 0}^{1}=\frac{\epsilon\left(\alpha_{J}-\beta_{r}\right)}{1+\alpha_{J}}, \\
& \tilde{v}_{t 1}^{1}=\frac{\epsilon}{b}\left[u_{t 0}+\frac{\epsilon \omega_{z 0}^{d} u_{20}}{1+\alpha_{J}+\epsilon\left(\beta_{r}-\alpha_{J}\right)}\right],
\end{aligned}
$$




$$
\begin{gathered}
\tilde{e}_{t 0}^{1}=\frac{-\epsilon\left(1+\alpha_{J}\right) \omega_{z 0}^{d}}{b\left[1+\alpha_{J}+\epsilon\left(\beta_{r}-\alpha_{J}\right)\right]} \quad(\operatorname{cgp} 1)(24 \mathrm{a}) \\
\tilde{v}_{A 1}^{2}=\frac{\epsilon}{1+\alpha_{J}}\left[u_{20}+\frac{\epsilon \alpha_{J} \omega_{z 0}^{d} u_{t 0}}{b}-\frac{\epsilon \alpha_{J}\left(\beta_{r} u_{10}+u_{30}\right)}{\left(1+\alpha_{J}\right)^{2}}\right] \\
\tilde{e}_{r 0}^{2}=\frac{\epsilon}{1+\alpha_{J}}\left[\alpha_{J}-\beta_{r}+\frac{\epsilon \alpha_{J}\left(\omega_{z 0}^{d}\right)^{2}}{b}-\frac{\epsilon \alpha_{J}\left(\alpha_{J}-\beta_{r}\right)^{2}}{\left(1+\alpha_{J}\right)^{2}}\right], \\
\quad+\frac{-\epsilon \omega_{z 0}^{d}}{b}+\frac{\epsilon^{2}}{b^{2}}\left[\left(\frac{b \alpha_{J}-1}{1+\alpha_{J}}+\beta_{t}-\frac{\beta_{z}}{\alpha_{I}}-\frac{(1+b) \beta_{r}}{1+\alpha_{J}}\right) \omega_{z 0}^{d}\right. \\
\tilde{e}_{t 0}^{2}=\left\{\frac{u_{I}}{\left(1-\tilde{e}_{r 0}^{2}\right)}\right. \\
\tilde{v}_{t 1}^{2}=\frac{\epsilon u_{t 0}}{b}+\frac{\epsilon^{2}}{b^{2}}\left[\frac{\left(u_{10}-b u_{20}\right) \omega_{z 0}^{d}}{1+\alpha_{J}}-\beta_{t} u_{t 0}\right]-\tilde{v}_{A 1}^{2} \tilde{e}_{t 0}^{2} . \\
(\mathrm{cgp} 2)(24 b)
\end{gathered}
$$

\section{Simulations}

Let us consider the inertial motion of sleigh under the case of $c \neq 0$ [3], i.e., the vertical noncoincidence of center $C$ and contact $A$ points. External force $E_{s}$ and control $u_{s}$ are not considered here. Complete and ideal equations of motion are

$$
\begin{gathered}
\dot{v}_{l}=v_{t} \omega_{z}+c \omega_{z}^{2}, \quad \dot{v}_{t}=-v_{l} \omega_{z}-\frac{I_{A} b_{T} v_{t}}{m I}, \quad \dot{\omega}_{z}=\frac{b_{T} c v_{t}}{I} \\
\text { (complete) }(25) \\
\dot{v}_{l}=c \omega_{z}^{2}, \quad v_{t}=0, \quad c K^{2} \dot{\omega}_{z}+v_{l} \omega_{z}=0 \quad \text { (ideal) (26) }
\end{gathered}
$$

where the inertial parameter $K^{2}=I_{A} /\left(m c^{2}\right)=1+$ $I /\left(m c^{2}\right)$. According to [3], the ideal sleigh has an asymptotic solution under $\left.v_{l}\right|_{t=0}=\left.v_{t}\right|_{t=0}=0$,

$$
y=x \tan \frac{K \pi}{2}+\text { constant }
$$

The ideal dash-dot results $(K=1,1.5,2,2.5)$ in Fig. 6 indeed behave such tendency.

The approximated quasi manifold $\tilde{\mathcal{M}}_{3}$ can be obtained as

$$
v_{t} \sim \epsilon\left(\frac{-m I v_{l} \omega_{z}}{I_{A}}\right)+\epsilon^{2} \frac{c m^{2} I^{2} \omega_{z}}{I_{A}^{2}}\left(\omega_{z}^{2}-\frac{m v_{l}^{2}}{I_{A}}\right)
$$

Hence, the dynamics of creep 1 and creep 2 are calculated. Creep 2 is simulated closer to the complete one than creep1. The simulation data are : $m=10$ $(\mathrm{Kg}), c=0.5(\mathrm{~m}), b_{x}=100(\mathrm{nt} \cdot \mathrm{sec} / \mathrm{m}), \epsilon=0.01$, $\left.\left(v_{1}, v_{t}, \omega_{z}\right)\right|_{t=0}=(0,0,1)(\mathrm{rad} / \mathrm{sec})$. All of them are assumed to stay inside a small viscous region.

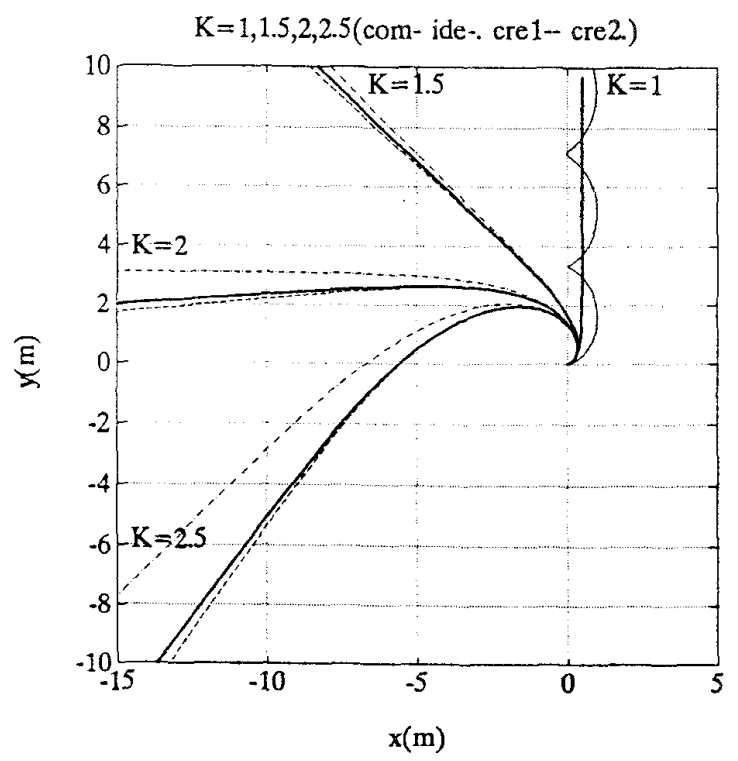

Fig. 6 Inertial motions of sleigh.

Next one is the rolling wheel. Related data are $m=1(\mathrm{Kg}), r=0.5(\mathrm{~m}), I=0.5\left(\mathrm{Kgm}^{2}\right), J=1$ $\left(\mathrm{Kgm}^{2}\right)$ and $\left.(x, y, \phi, \theta)\right|_{t=0}=(0,0,0,0),\left.v_{l}\right|_{t=0}=1$ $(\mathrm{m} / \mathrm{sec}),\left.v_{t}\right|_{t=0}=0,\left.\omega_{r}\right|_{t=0}=2(\mathrm{rad} / \mathrm{sec}), v_{A U}=0.1$ (m/sec), and $f_{R U}=f_{T U}=1$ (nt). Fig. 7 shows the $x-y, e_{r}$ and $e_{t}$ results of hybrid creep dynamics and creep of general-pair. Both them prove the validity of bridge relations.

The $10 \mathrm{~Hz}$ braking torque $u_{r}$ in an ABS and the 20 $\mathrm{Hz}$ steering $\omega_{z}^{d}$ in a special TCS in Fig. 8 are used to demonstrate the applications of creep. Fig. 9 shows that $v_{A}$ and $v_{t}$ can only be limited within a small region under the action of ABS or TCS. Otherwise, slipping will occur and it is dangerous.

\section{Conclusions}

The proposed creep model can be suitably used to account for most quasi constrained behaviors. By the creep parameters, the pseudo violated speeds could be approximated as close as possible even under a kinematical motion analysis without considering the system dynamics. This is the core contribution of creep analysis. 


\section{References}

[1] J.W. Kamman, R.L. Huston, "Dynamics of Constrained Multibody Sys- tems," ASME J. of Applied Mechanics, Vol. 51, pp. 899-903, 1984.

[2] A.V. Karapetian, "On Realizing Nonholonomic Constraints by Viscous Friction Forces and Celtic Stones Stability," PMM, U.S.S.R., Vol. 45, pp. 30-36, 1982.

[3] J.I. Nelmark, N.A. Fufaev, Dynamics of Nonholonomic Systems, American Mathematical Society, Providence, Rhode Island, 1972.

[4] V.A. Sobolov, "Integral Manifolds and Decomposition of Singularly Perturbed Systems," Systems and Control Letters, Vol. 5, pp. 169-179, 1984.

[5] J.C. Wang, H.P. Huang, "Creep Kinematics of Nonholonomic Systems," IEEE Intl. Conf. on Robotics and Automation, Nagoya, Japan, 1995.

[6] J.C. Wang, Geometry, Kinematics, Dynamics and Control for Quasi Constrained Mechanical Systems, Ph. D. Dissertation, Mech. Eng., Nat. Taiwan Univ., Taiwan, 1995.
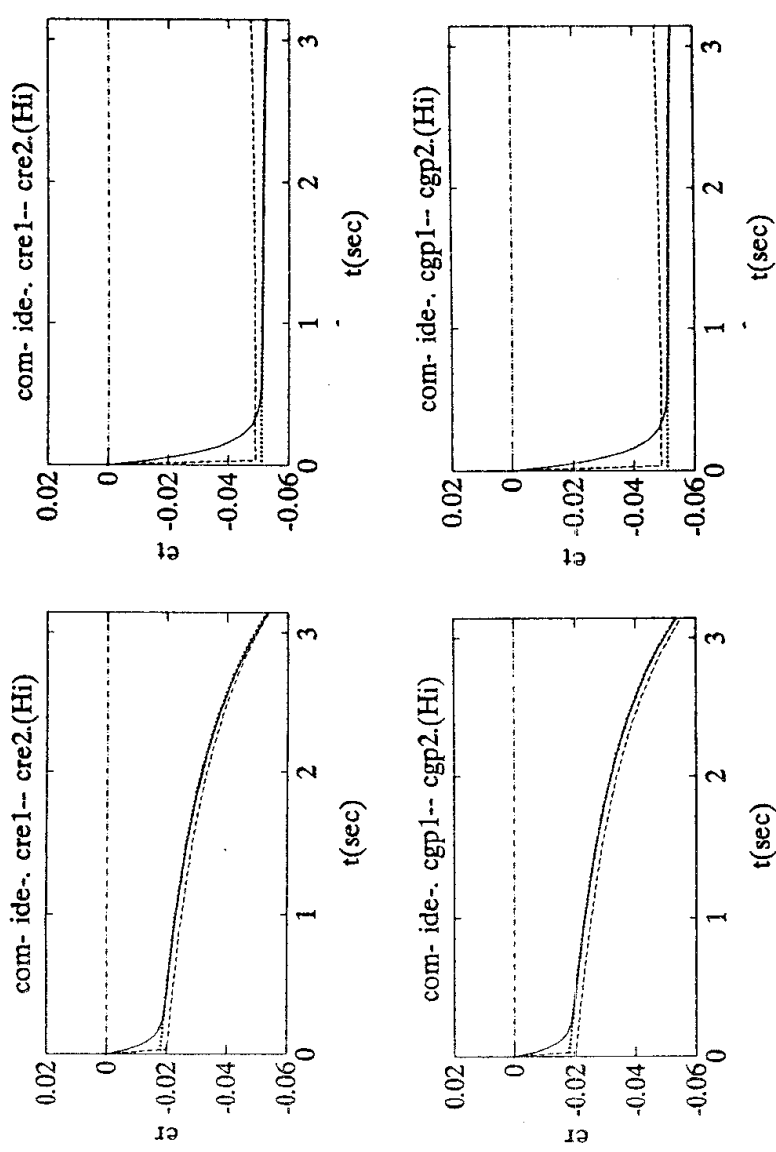

Fig. 7 Hybrid creep and general-pair simulations.

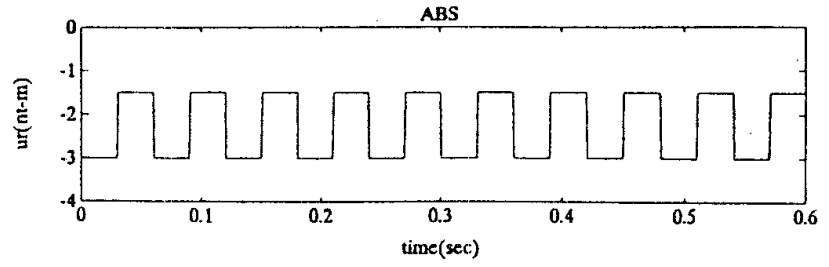

TCS

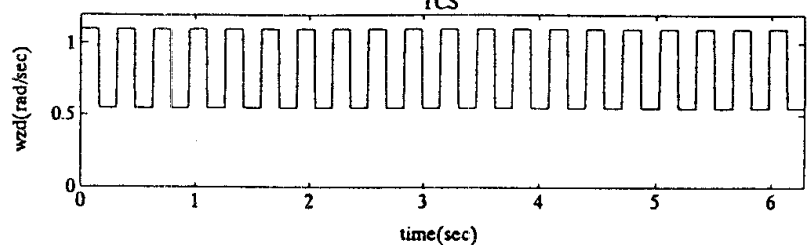

Fig. 8 ABS and TCS control torques.
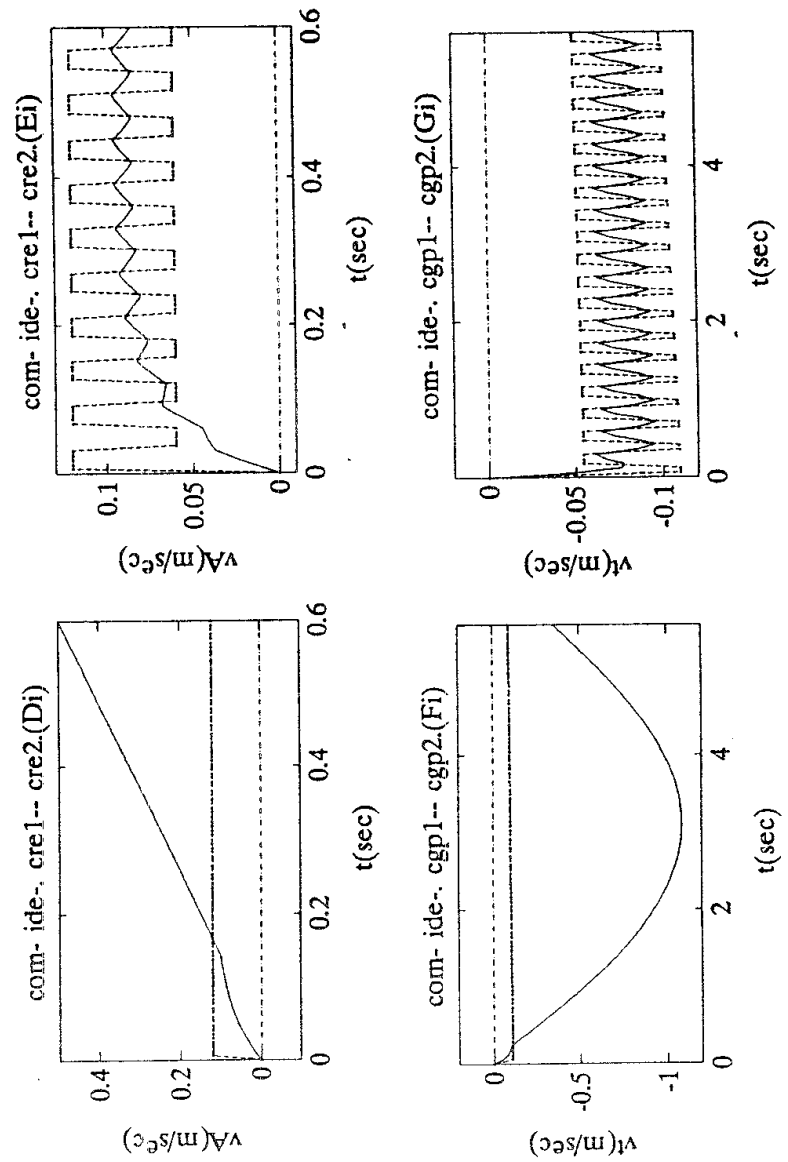

Fig. \& Simulations of ABS (cre) and TCS (cgp). 